\title{
General Psychiatry Different levels of facial expression recognition in patients with first- episode schizophrenia: A functional MRI study
}

Chengqing Yang, ${ }^{1}$ Ansi Qi, ${ }^{2}$ Huangfang Yu, ${ }^{3}$ Xiaofeng Guan, ${ }^{2}$ Jijun Wang, ${ }^{1} \mathrm{Na} \mathrm{Liu},{ }^{1}$ Tianhong Zhang, ${ }^{1}$ Hui Li, ${ }^{1}$ Hui Zhou, ${ }^{1}$ Junjuan Zhu, ${ }^{1}$ Nan Huang, ${ }^{1}$ Yingying Tang, ${ }^{1}$ Zheng Lu ${ }^{1,2}$
To cite: Yang C, Qi A, Yu H, et al. Different levels of facial expression recognition in patients with first-episode schizophrenia: A functional MRI study. General Psychiatry 2018;31:e000014. doi:10.1136/ gpsych-2018-000014

- Prepublication history for this paper is available online. To view these files, please visit the journal online (http://dx.doi.org/ 10.1136/gpsych-2018-000014)

Received 20 November 2017

Revised 26 April 2018

Accepted 07 May 2018

\section{Check for updates}

(C) Author(s) (or their employer(s)) 2018. Re-use permitted under CC BY-NC. No commercial re-use. See rights and permissions. Published by BMJ.

${ }^{1}$ Shanghai Mental Health Center, Shanghai, China

${ }^{2}$ Tongji Hospital Affiliated to Tongji University, Shanghai, China

${ }^{3}$ Jinshan Hospital Affiliated to Fudan University, Shanghai, China

Correspondence to

Zheng Lu;

luzheng@tongji.edu.cn

\section{ABSTRACT}

Background The impairment of facial expression recognition has become a biomarker for early identification of first-episode schizophrenia, and this kind of research is increasing.

Aims To explore the differences in brain area activation using different degrees of disgusted facial expression recognition in antipsychotic-naïve patients with firstepisode schizophrenia and healthy controls.

Methods In this study, facial expression recognition tests were performed on 30 first-episode, antipsychoticnaïve patients with schizophrenia (treatment group) and 30 healthy subjects (control group) with matched age, educational attainment and gender. Functional MRI was used for comparing the differences of the brain areas of activation between the two groups.

Results The average response time difference between the patient group and the control group in the 'high degree of disgust' facial expression recognition task was statistically significant (1.359 (0.408)/2.193 (0.625), $\mathrm{F}=26.65, \mathrm{p}<0.001)$, and the correct recognition rate of the treatment group was lower than that of the control group (41.05 (22.25)/59.84 (13.91, $F=19.81, p<0.001)$. Compared with the control group, the left thalamus, right lingual gyrus and right middle temporal gyrus were negatively activated in the patients with first-episode schizophrenia in the 'high degree of disgust' emotion recognition, and there was a significant activation in the left and right middle temporal gyrus and the right caudate nucleus. However, there was no significant activation difference in the 'low degree of disgust' recognition. Conclusions In patients with first-episode schizophrenia, the areas of facial recognition impairment are significantly different in different degrees of disgust facial expression recognition.

\section{BACKGROUND}

Schizophrenia is a severe mental disorder. The causes are still unknown and early diagnosis is relatively difficult. The onset of this disorder commonly begins in early adulthood and peaks around the age of 20 to 24 for men and 29 to 32 for women. Patients usually have multidimensional problems in perception, thoughts, emotion and behaviour, accompanied by cognitive impairment. Much research ${ }^{12}$ has already found that patients with schizophrenia had cognitive impairment, and this cognitive impairment is comparatively independent of the positive and negative symptoms of schizophrenia. Cognitive deficits are closely related to the social functioning of patients with schizophrenia. Current studies suggest that cognitive function is one of the critical predictive factors of the recovery of social functioning in patients with schizophrenia. ${ }^{3}{ }^{4}$ Facial expression recognition is a part of social cognition. The ability to recognise facial expressions plays an important role in people's daily lives. Some PET and functional MRI studies related to facial expression recognition have shown that there is an activation in the fusiform gyrus of the brain when patients with schizophrenia perform facial recognition of fear. The current study suggests that the fusiform gyrus region is a functional area of specificity for facial expression recognition ${ }^{3-6}$; however, the study results of activation of local regions in the brain induced by other expressions were inconsistent. ${ }^{5-11}$ In particular, there were relatively few functional MRI studies on varying degrees of facial expression recognition. ${ }^{12-15}$ The facial expression of disgust, as the basic facial expression, has increasingly received the attention of scholars. This research used functional MRI, taking patients with first-episode schizophrenia who have never had antipsychotic medication and healthy controls as the study subjects, to primarily observe the similarities and differences between the brain activation regions when recognising different degrees of a disgusted facial expression. 


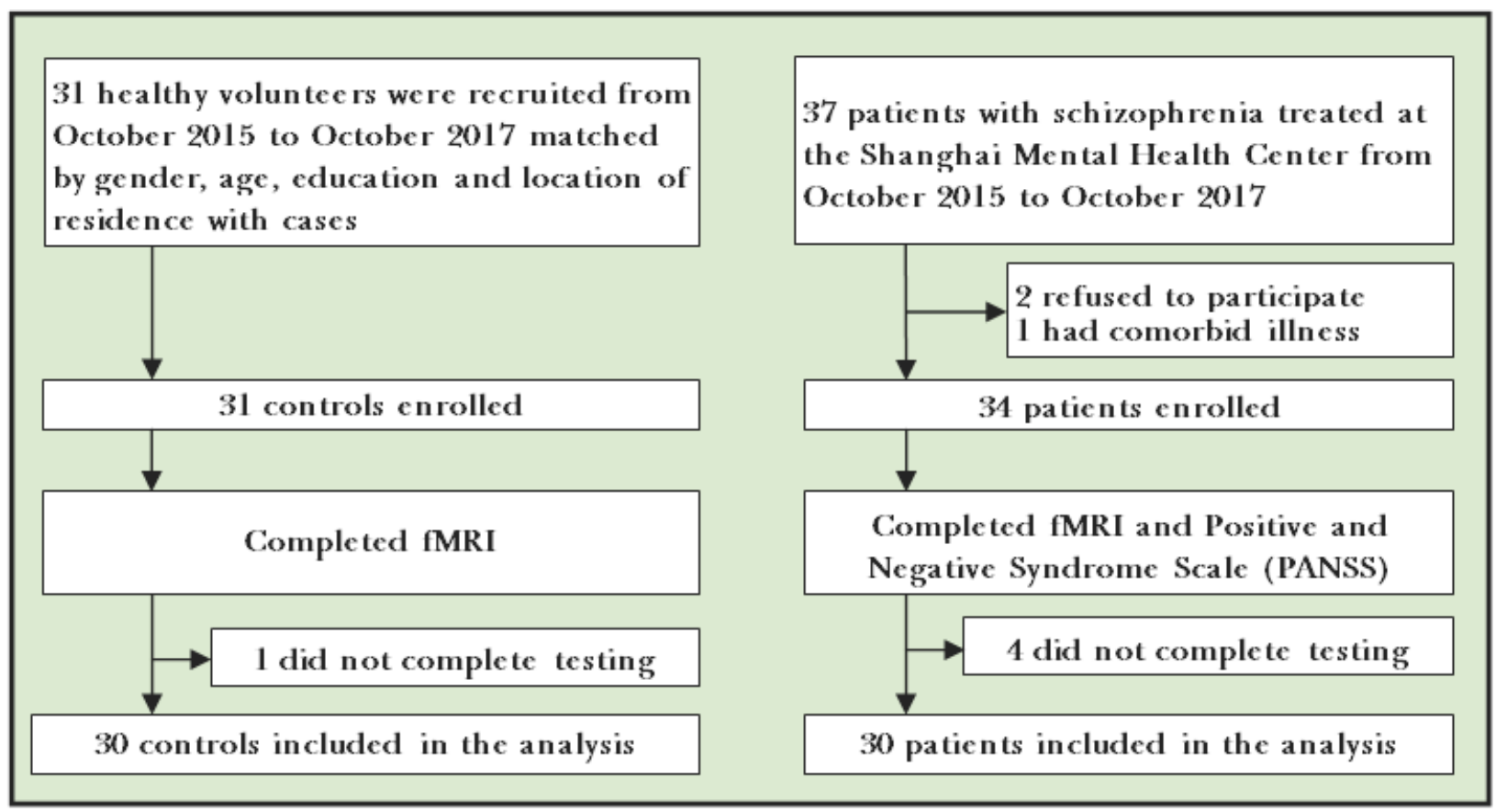

Figure 1 Flowchart of the study.

\section{PARTICIPANTS AND METHODS}

This project took place from January 2015 to November 2017.

\section{Participants}

Treatment group met the following inclusion criteria: (1) met the diagnostic criteria for schizophrenia or schizophrenia-like psychosis according to the Diagnostic and Statistical Manual of Mental Disorders fourth edition; (2) aged from 18 to 30 years old, Han ethnicity, right handed; (3) first episode, course of disease $<2$ years, had never taken antipsychotics or stopped antipsychotics for five half-life periods or more; (4) the IQ of the Wechsler Intelligence Scale was $>70$. The exclusion criteria were the following: (1) having contraindications to MRI; (2) mental retardation and nervous system diseases, major somatic diseases; (3) history of brain trauma, indication of abnormal brain structure by MRI examination; (4) history of alcohol or drug abuse or dependence; (5) received Modified Electra Convulsive Therapy (MECT) and long-term use of medication such as anticholinergics and benzodiazepine that could potentially affect cognitive function.

In this study, 37 patients with schizophrenia were recruited (see figure 1). Among those patients, two cases declined to participate in the study, one case was excluded due to a change of diagnosis to mental disorders due to systemic lupus erythematosus that occurred approximately 1 month after hospitalisation; four patients with schizophrenia were not cooperative during the MRI examination; therefore, 30 patients were successfully enrolled. Among these 30 patients, 14 were men and 16 were women, and the mean (SD) age of this group was 25 (7) years. Educational attainment for this group was the following: junior high school, 4 cases; senior high school or technical secondary school, 13 cases; University or graduate school, 5 cases. The patients scored between 4 and 6 on the Clinical Global Impression Scale (CGI) during the psychiatric examination. ${ }^{16}$ In this study, two senior associate chief physicians of the Shanghai Mental Health Center conducted an evaluation of relevant clinical information on patients with first-episode schizophrenia, including the evaluation for psychotic symptoms using the Positive and Negative Syndrome Scale (PANSS). The Kappa value of the consistency test by the two scale raters was 0.85 .

The control group had the following inclusion criteria: (1) aged from 18 to 30 years, Han ethnicity and right handed; (2) IQ on the Wechsler Intelligence Scale was $>70$; (3) not having a psychotic disorder. Exclusion criteria were the following: (1) contraindications for having an MRI; (2) history of mental illness and a family history of major psychosomatic disorders; (3) brain trauma, or indication of abnormal brain structure by MRI; (4) alcohol or drug abuse. In this study, 31 healthy controls were recruited. There was one participant in the control group who had constant head movement during the MRI, which resulted in an incomplete examination. Therefore, the final number of enrolled controls was 30. In this study, all healthy controls enrolled were right handed. The two groups were matched for gender, age and educational level. See table 1 for details.

\section{Study methods}

The examination was conducted in the MRI room at the Shanghai Mental Health Center. The facial expressions adopted in this experiment were selected from the German Ekman standard black and white facial 
Table 1 Demographic information for participants

\begin{tabular}{|c|c|c|c|c|}
\hline Items & $\begin{array}{l}\text { Healthy control group }(n=30) \\
\text { Mean (SD) }\end{array}$ & $\begin{array}{l}\text { Patient group }(n=30) \\
\text { Mean (SD) }\end{array}$ & $t / \chi^{2}$ & P values \\
\hline Gender & $16 \mathrm{~F} / 14 \mathrm{M}$ & $14 \mathrm{~F} / 16 \mathrm{M}$ & 0.267 & 0.606 \\
\hline Age & $27.258(5.791)$ & $25.622(7.786)$ & 1.824 & 0.074 \\
\hline Educational attainment & $12.62(0.389)$ & $12.86(0.458)$ & -0.648 & 0.519 \\
\hline \multicolumn{5}{|l|}{ PANSS } \\
\hline Positive & & $28.7(6.7)$ & & \\
\hline Negative & & $23.9(8.4)$ & & \\
\hline General & & $37.6(8.9)$ & & \\
\hline CGI & & $4.7(1.1)$ & & \\
\hline
\end{tabular}

${ }^{*} \mathrm{P}<0.001$.

CGI, Clinical Global Impression Scale; F, female; M, Male; PANSS, Positive and Negative Syndrome Scale.

expression library. The neutral facial expressions were obtained from an average of 20 adult men and women. The major selected facial expression in this experiment was disgust and the neutral facial expression was used for controls. The expression level of the pre-set neutral facial expression was $0 \%$, whereas the maximum setting of the facial expression level in the Ekman standard photo gallery was $100 \%$. This study used Morph Software to generate nine faces with expression levels from $10 \%$ to $90 \%$ in neutral facial expression and disgust expression, respectively; therefore, each facial expression had 44 faces (4 persons $\times 11$ faces). Each facial expression was divided into two levels: $10 \%$ to $50 \%$ as low level and $51 \%$ to $100 \%$ as high level. During the experiment, each facial expression was repeated three times. As a result, there were 132 faces in each facial expression test. The exposure time of each face was $400 \mathrm{~ms}$ and the black screen time was $1600 \mathrm{~ms}$. The subjects were requested to distinguish between each newly presented facial expression. The experiment was set up so participants pressed the ' 0 ' number key for neutral facial expression and the ' 3 ' number key for a disgusted facial expression. A $5 \mathrm{~min}$ practice was given to the subjects before the formal test. This was for the purpose of familiarising themselves with the experimental procedures and reaction of pressing the key. Details of the schematic of the mixed blocked/ event-related design can be seen in figure 2.

The specifics of the picture sample taking in the facial perception task were the following. For imaging parameters, the Siemens 3.0T MAGNETOM Trio Tim MRI scanner was used for performing brain MRI examination on the subjects. The scan content was the task-state scan. As a result of the high noise during the process of the scan, foam ear plugs were provided to each participant for noise reduction. In order to reduce the head movement of the subjects, a foam pad was placed between the head of the subjects and the coil. The repetition time was $(\mathrm{TR})=2530$, echo time $(\mathrm{TE})=3.65 \mathrm{~ms}$, flip angle $=70$ degrees, slice thickness $=1 \mathrm{~mm}$, field of view $($ FOV $)=256 \times 256 \mathrm{~mm}^{2}$, matrix $=256 \times 256$ and number of layers $=224$ layers. The participants received follow-up scans when no abnormal structures were found by the brain MRI scan. The functional MRI adopted echo-planar imaging for blood oxygen level dependent, $\mathrm{TR}=2000 \mathrm{~ms}$, $\mathrm{TE}=30 \mathrm{~ms}, \mathrm{FOV}=200 \times 200 \mathrm{~mm}$, flip angle $=90$ degrees, matrix $=74 \times 74$, voxel size $=3 \times 3 \times 3$ and 50 layers continuous scan, layer thickness $3 \mathrm{~mm}$.

\section{Statistical analysis}

Group t-tests were used for behavioural data. Postprocessing of functional imaging adopted spm12. At first, head motion correction was conducted followed by manually matching the functional images to structural images. Subsequently, the MRI images were segmented into grey matter and white matter and projected into the standardised space using the non-linear method. The voxel values of each subject in each brain region were calculated. In the end, smoothing processing was performed on the images, thus increasing the normality of data and achieving higher signal:noise ratio of the image. Initially, we conducted an individual-level analysis and compared the brain function activity levels in the correct emotion

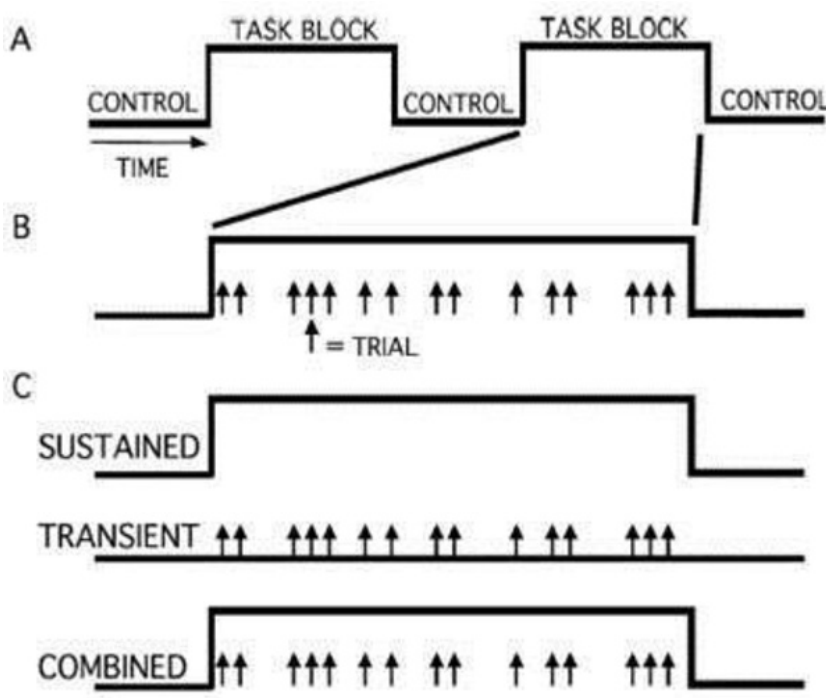

Figure 2 Canonical diagram: schematic of the mixed blocked/event-related design. 
judgement trials. The .con document obtained from the body-level analysis was used in the intragroup analysis. The one-sample t-test was used to compare intragroup differences of brain activation in subjects; analysis of variance was used to compare the diencephalon activation difference between the two groups, removing the effects of age and gender, voxel level uncorrected $\mathrm{p}<0.01$, consecutive prime number $>10$.

\section{RESULTS}

\section{Comparison of demographic data}

This study enrolled 30 patients with schizophrenia and 30 healthy controls. There were no statistically significant differences of gender, age and educational level between the two groups (see table 1).

\section{Behavioural data}

The reaction time of the two groups only measured the average reaction speed in which participants pressed the key. For specific results, see table 2. The difference in average reaction time for the high-level disgust facial expression recognition between the two groups was statistically significant. Moreover, the reaction time of the case group was slower than the control group, and the accuracy was also lower than the control group. There was no significant difference in the average reaction time and accuracy between the two groups in the low-level facial expression recognition $(\mathrm{F}=0.031, \mathrm{p}=0.861$ and $\mathrm{F}=2.225$, $\mathrm{p}=0.141$, respectively).

\section{Comparison of the functional MRI results}

In comparing facial expression recognition to the highlevel disgust expressions between the two groups, activation was only reduced in three regions (see figure 3): the left thalamus, the right lingual gyrus and the right middle temporal gyrus. Between the disgust emotion and neutral emotion, the left and right middle temporal gyrus and the right caudate nucleus had significant activation. However, there was no significant difference in regional activation between the two groups when performing facial expression recognition on the low-level disgust expression. See table 1 for details.

\section{DISCUSSION}

\section{Main findings}

This test used an internationally recognised standardised facial expression database (Ekman facial expression library) to carry out research on the recognition of disgusted facial expressions with varying degrees in drugnaive patients with first-episode schizophrenia. Results showed that there was brain activation impairment on the high-level facial perception in the drug-naive patients with first-episode schizophrenia from the perspective of functional neuroimaging. In the high-level disgust expression recognition, the reaction time of the patient group was longer than the control group and the reaction accuracy rate was apparently lower in the control group as well. This suggests that there was a delay in response and decrease in judgement in patients with schizophrenia. In addition, a significant difference was not found for the recognition of low-level disgust between the two groups, which may indicate that patients with schizophrenia are not sensitive to expressions of a low level of disgust. Moreover, the activation of the left thalamus in the patients with schizophrenia in this study was lower than that in the control group. The thalamus, which is the relay station of the brain, acts as a filter, receives all kinds of information and excludes redundant or irrelevant stimuli so as to transmit important information. This indicates that the defect in information filtering in patients with schizophrenia could be related to selected cognitive tasks. This study also found that the activation of the right lingual gyrus and right middle temporal gyrus of the patient group was weaker than the normal group when the subjects of the two groups performed facial recognition tasks. Based on the existing research, it is clear that there is a difference in the brain network during the facial expression recognition tasks when comparing patients with schizophrenia and healthy individuals. It could be that there is some association in the prefrontal cortex, occipital lobe and several areas of the temporal lobe (fusiform gyrus, right lingual gyrus, right middle temporal gyrus, temporal pole, amygdala and hippocampus) through different modes of the magnetic resonance research cross-validation; however, the exact brain

Table 2 Comparison of the reaction time and accuracy rate between the two groups across varying levels of disgusted facial expression

\begin{tabular}{|c|c|c|c|c|}
\hline Items & $\begin{array}{l}\text { Patient group } \\
\text { Mean (SD) }\end{array}$ & $\begin{array}{l}\text { Control group } \\
\text { Mean (SD) }\end{array}$ & $\mathbf{F}$ & P values \\
\hline \multicolumn{5}{|l|}{ Low level } \\
\hline Reaction time & 2120.910 (710.903) & $1872.508(662.220)$ & 0.031 & 0.861 \\
\hline \multicolumn{5}{|l|}{ High level } \\
\hline Accuracy rate & $0.4105(0.222)$ & $0.598(0.139)$ & 19.81 & $<0.001$ \\
\hline
\end{tabular}




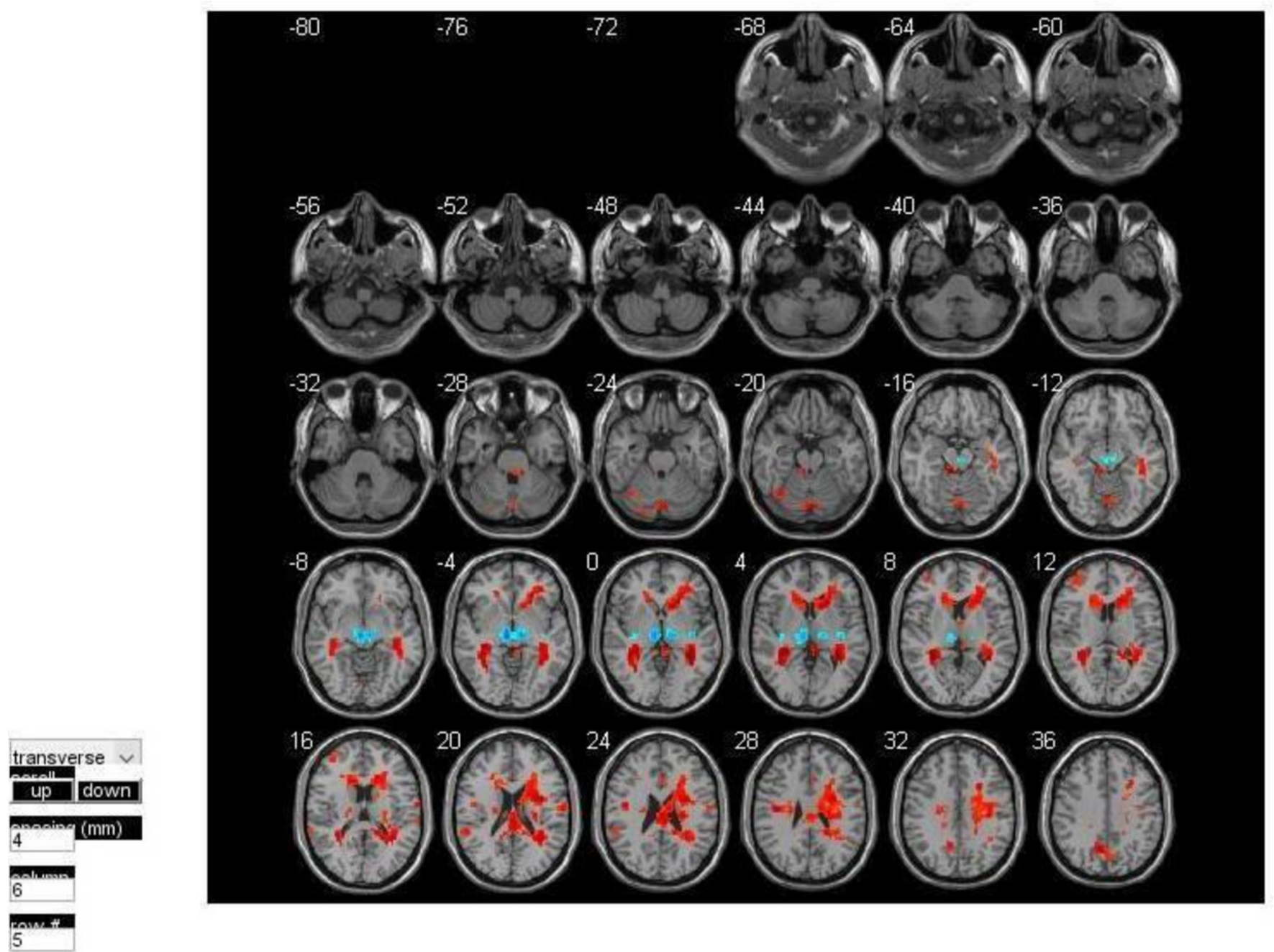

Figure 3 Difference in brain activation sites of the two groups in the facial perception task.

network mechanism is unknown. ${ }^{17}$ Other studies have also found that the amygdala response in patients with schizophrenia was significantly higher than in healthy controls during perception of sadness and happiness, and this enhancement was positively correlated with the negative symptoms of the PANSS. ${ }^{18}$ In the determination of fear or neutral emotion, it was found that the correlation was high only in the occipital lingual gyrus and superior temporal gyrus. ${ }^{19}$ Fakra and colleagues ${ }^{20}$ divided facial expression recognition into matching and labelling and found that there was no significant difference between the schizophrenia group and normal control group in the labelling task. During matching tasks, patients with schizophrenia showed decreased activation of the limbic system and lack of connectivity between the amygdala and prefrontal cortex.

\section{Limitations}

This study had a small sample size. Although the Ekman facial expression library is the standard facial expression library, the sources of the main facial expression were foreigners. In the future, a Chinese facial expression library can be established to increase the reliability of the experimental result. Second, the activation criteria used in the statistical method (voxel level uncorrected $\mathrm{p}<0.01$, continuous voxel number $>10)$ are basically experiences and attempts; therefore, further tests and assessments are needed in future studies. Lastly, this study did not have further follow-up with patients. Future studies could conduct follow-up interviews with participants.

\section{Implications}

Studies of basic emotions suggest that the emotional processing during facial expression recognition of drugnaive patients with first-episode schizophrenia is different from that of healthy controls. There may be disturbances in the network of emotional regulation in the occipital lobe, right lingual gyrus, right middle temporal gyrus, anterior temporal pole, amygdala and prefrontal cortex. ${ }^{21}$ Previous studies of healthy individuals ${ }^{21}$ found that these brain regions corresponded to the 'society-brain' face recognition network in the prefrontal cortex- basal ganglia of ventral temporal lobe. Similarly, the first-degree relatives of those with schizophrenia also had an abnormal brain network in this region. Further studies can be carried out with first-degree relatives of those with schizophrenia to facilitate early identification of the disease and exploration of the pathogenesis. 
Acknowledgements The collection of volunteer faces in this study was supported by Professor Sun Yaoru and Dr Zhang Zuo of the School of Telecommunications, Tongji University, Shanghai.

Contributors $\mathrm{YC}$ was responsible for writing and revising the article, as well as the experiment design and statistical methods. QA was responsible for participant enrollment. YH was responsible for enrolment of healthy controls. GX was responsible for participant enrolment. WJ was responsible for experiment design. NL was responsible for participant enrolment and quality control. ZT was responsible for the experiment design and the guidance on statistical methods. LH was responsible for the experiment design and functional magnetic resonance data processing methods. ZH was responsible for participant enrolment. ZJ was responsible for participant enrolment. HN was responsible for participant enrolment. TY was responsible for the experiment design. LZ was responsible for the participant enrolment and the management and quality control of the research process.

Funding Shanghai Mental Health Center hospital-level research project (2016YJ-04), National Key Technology R\&D Program of China during the 10th Five-Year Plan Period (2007BAl17B04), National Key Research and Development Program (2016YFC1306805), National Natural Science Foundation of China (81471359), Shanghai Municipal Committee of Science and Technology Guide Project of Chinese and Western Medicine (14411963400).

Competing interests None declared.

Patient consent Obtained.

Ethics approval This study was approved by the ethics committee of the Shanghai Mental Health Center.

Provenance and peer review Not comissioned; externally peer reviewed.

Data sharing statement No additional data are available.

Open access This is an open access article distributed in accordance with the Creative Commons Attribution Non Commercial (CC BY-NC 4.0) license, which permits others to distribute, remix, adapt, build upon this work non-commercially, and license their derivative works on different terms, provided the original work is properly cited and the use is non-commercial. See:http://creativecommons.org/ licenses/by-nc/4.0

\section{REFERENCES}

1. Goldberg TE, Weinberger DR. Effects of neuroleptic medications on the cognition of patients with schizophrenia: a review of recent studies. J Clin Psychiatry 1996;57(Suppl 9):62-5.

2. Green MF, Kern RS, Heaton RK. Longitudinal studies of cognition and functional outcome in schizophrenia: implications for MATRICS. Schizophr Res 2004;72:41-51.

3. Mueser KT, Doonan R, Penn DL, et al. Emotion recognition and social competence in chronic schizophrenia. J Abnorm Psychol 1996;105:271-5.
4. Chambon V, Baudouin JY, Franck N. The role of configura information in facial emotion recognition in schizophrenia. Neuropsychologia 2006;44:2437-44.

5. Yang CQ, Lu Z, Wang JJ. Research in facial recognition examined by event-related potentials in patients with the first-episode schizophrenia. Zhong Hua Jing Shen Ke Za Zhi 2012;45:74-9.

6. Fett AK, Maat A, GROUP Investigators. Social cognitive impairments and psychotic symptoms: what is the nature of their association? Schizophr Bull 2013;39:77-85.

7. Sui J, Yu Q, He H, et al. A selective review of multimodal fusion methods in schizophrenia. Front Hum Neurosci 2012;6:27.

8. He Y, Chen Z, Gong G, et al. Neuronal networks in Alzheimer's disease. Neuroscientist 2009;15:333-50.

9. Sheng J, Zhu Y, Lu Z, et al. Altered volume and lateralization of language-related regions in first-episode schizophrenia. Schizophr Res 2013;148:168-74.

10. Goghari VM, Macdonald AW, Sponheim SR. Temporal lobe structures and facial emotion recognition in schizophrenia patients and nonpsychotic relatives. Schizophr Bull 2011;37:1281-94.

11. Roalf DR, Ruparel K, Verma R, et al. White matter organization and neurocognitive performance variability in schizophrenia. Schizophr Res 2013;143:172-8.

12. Lee J, Gosselin F, Wynn JK, et al. How do schizophrenia patients use visual information to decode facial emotion? Schizophr Bull 2011;37:1001-8.

13. Morris RW, Weickert CS, Loughland CM. Emotional face processing in schizophrenia. Curr Opin Psychiatry 2009;22:140-6.

14. Eack SM, Mermon DE, Montrose DM, et al. Social cognition deficits among individuals at familial high risk for schizophrenia. Schizophr Bull 2010;36:1081-8.

15. Lynall ME, Bassett DS, Kerwin R, et al. Functional connectivity and brain networks in schizophrenia. $J$ Neurosci 2010;30:9477-87.

16. Liu $\mathrm{H}$, Liu Z, Liang $\mathrm{M}$, et al. Decreased regional homogeneity in schizophrenia: a resting state functional magnetic resonance imaging study. Neuroreport 2006;17:19-22.

17. Berk M, Ng F, Dodd S, et al. The validity of the CGI severity and improvement scales as measures of clinical effectiveness suitable for routine clinical use. J Eval Clin Pract 2008;14:979-83.

18. Rauch AV, Reker M, Ohrmann P, et al. Increased amygdala activation during automatic processing of facial emotion in schizophrenia. Psychiatry Res 2010;182:200-6.

19. Leitman DI, Loughead J, Wolf DH, et al. Abnormal superior temporal connectivity during fear perception in schizophrenia. Schizophr Bull 2008;34:673-8.

20. Fakra E, Salgado-Pineda P, Delaveau P, et al. Neural bases of different cognitive strategies for facial affect processing in schizophrenia. Schizophr Res 2008;100:191-205.

21. Li H, Chan RC, McAlonan GM, et al. Facial emotion processing in schizophrenia: a meta-analysis of functional neuroimaging data. Schizophr Bull 2010;36:1029-39.

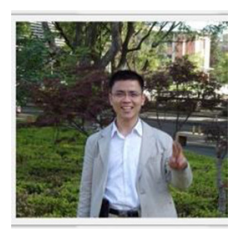

Chengqing Yang graduated from Tongii University School of medicine in 2003 and received his master's degree in 2011. In 2008, he began to work and study at the Shanghai Mental Health Center. He is currently working as an attending physician. His research directions are mainly schizophrenia, ERP, and fMRI. 